\title{
Is Indian Cancer Unique?
}

\author{
Chintamani $^{1}$
}

Published online: 13 November 2015

(C) Association of Surgeons of India 2015

There is nothing more deceptive than an obvious fact.

.... Arthur Conan Doyle

That Indian cancers are different from their Western counterparts is a hotly debated issue in most cancer meetings and often there is a tendency to use this argument to explain our inability to manage this epidemic optimally. The evidence is however otherwise; cancer respects no boundaries or barriers, although Indian circumstances could be different. It has been observed that certain cancers do show some predilection for a particular race e.g Indian Fijian women immigrants developing gall bladder cancer more often than their local counter parts. However, by and large, as the immigrants settle down and as the second or third generation adapts to the local life style, these differences mostly disappear [1-4].

\section{Magnitude of Indian Problem}

According to one World Health Organization report, cancer is rapidly sweeping through India with nearly one cancer happening every 50 seconds. The problem is taxing and stressing a health care system that is already overburdened by the world's sickest people. Hundreds of thousands need surgery for cancer and years of treatment drive a quarter of these households into poverty. According to the World Bank, India will lose nearly " $\$ 20$ billion in economic output from 2012 to 2030 as a result

Chintamani

drchintamani7@gmail.com

1 Delhi, India

of breast cancer alone". It is therefore not even smart economics to ignore this emerging and serious health problem [3].

\section{The Changing Indian Scenario}

It has been observed in the last decade that most Indian cancers are diagnosed at a relatively younger age (mean age having dropped by 5 years). These cancers are usually more advanced at presentation and also biologically more aggressive. Breast cancer, which is the most common cancer of the human race, is fast becoming the most common cancer amongst Indian women replacing cancer of the cervix, especially in the metros. Sadly, nearly $60 \%$ cancer patients in India die in the first year of diagnosis, as only about $30 \%$ are curable when diagnosed. Adding insult to injury is the fact that half of these patients receive treatment from generalists and not from cancer specialists.

\section{We Are Treating the Same Disease, Only the Circumstances Are Different!!}

People almost invariably arrive at their beliefs not on the basis of proof but on the basis of what they find attractive.

...Blaise Pascal, De l'art de persuader

\section{Younger Age at Presentation}

At present, the majority of Indian population is young and this could well explain this phenomenon. The 
increasing life expectancy has also contributed to the overall increased incidence, which is also reflected in the Indian contribution to the global figures. Nearly $17 \%$ of the world population lives in India, which is home to $21 \%$ of global cancer burden. In a study published by author and his group in the year 2005, two peaks of breast cancer incidence were observed (one in 40s and the other in 60s) unlike in the West. On revisiting this data (with more cases included in the last decade), the patterns are almost matching those observed in the West $[1,2]$.

\section{Advanced Stage at Presentation}

This is not very difficult to understand in a country where health gets a very low priority. The delayed diagnosis and advanced stage at presentation are often blamed on fatalistic attitudes, low socioeconomic status and low literacy rates of Indian masses, but the author (in one of his published series) observed that the delays were more often due to providers. These delays were alarming in some cases as they happened in some tertiary care training centres [3].

There are very few national cancer screening programmes and minimal involvement of state in the health of its people. As a contrast, screening in the West has been found to significantly reduce mortality especially in breast cancers by detecting them early. There are various issues with these programmes in India, the major being the cost involved and the fact that our average tumour size is still on the larger side. We would instead need to evolve programmes to increase cancer awareness and use some inexpensive methods (dermatoglyphics etc.) to get them early [4]. In the name of increasing awareness about cancer, we do have some state health programmes that have yielded too little to write home about.

\section{How Have We Done So Far?}

"According to Harvard School of Public Health Boston projects on Cancer Survival and WHO data, our five year survival rates for breast cancer are meager $52 \%$ as compared to $82 \%$ in China and $89 \%$ in US. Cancer is a bigger killer in India than AIDS, malaria and tuberculosis combined. It has been observed that more than $50 \%$ new cases of cancer and more than $2 / 3 \mathrm{rd}$ of cancer deaths (as compared to $15 \%$ in 1970) occur in India while less than $5 \%$ of global spending on cancer is done in this part of the world" (Lancet 2010). We are therefore sitting on a ticking time bomb that is waiting to explode and our response and preparation for the challenge is not inspiring to say the least.

\section{Seeing into the Future!!}

One can observe striking similarities of present-day Indian cancer patterns with those of the developed world in 1960s and 1970s. Studying cancer patterns in Indian immigrants to various developed nations (when compared with the local population back home) can serve as a useful tool to look into the future to plan management strategies for India by predicting the patterns as urbanization and Westernization continue. It has been observed in various studies that with immigrants adapting the local culture, lifestyle and dietary habits, the cancer incidence patterns gradually change towards that of the host country. Thus, environment and lifestyle changes come across as more significant factors rather than race and the country of origin. This should be good news as these factors can be managed if we plan well and in advance. The sudden spurt in cancer cases in India in last couple of decades has a definite association with the increasing weternization and "burger cola culture" imported in the recent past $[3,4]$.

\section{Finding Indian Solutions!}

....and when is enough proof enough? ...Jonathan

Safran Foer, Everything is illuminated

There is no such entity as an Indian cancer; we need to follow the global cancer guidelines with Indian scenario in mind. We have to find "our" solutions to this "global" problem and our job is cut out if we intend to prepare ourselves for this imminent disaster. We also need to learn from mistakes committed in the West in the name of "development" in order to find a middle path with minimal deviation from our original Indian lifestyle. India continues to be a land of paradoxes, possessing some health care centres that can match the best in the world while the nation is still struggling to provide primary health care to the majority population. In the name of development, we should not merely succeed in replacing communicable diseases, the present-day killers, with this killer of the twenty-first century. We need to work towards its prevention, early detection and optimum management by educating and increasing awareness about cancer not only amongst the masses but also the health care providers. As India progresses from a developing to a developed country, we should not end up creating a monster that in real sense makes this progress futile or a proverbial run on a tread mill. 


\section{References}

1. Saxena S, Rekhi B, Bansal A, Bagga A, Chintamani, Murthy NS (2005) Clinico-morphological patterns of breast cancer including family history in a New Delhi hospital, India - a cross-sectional study. World J Surg Oncol 3:67

2. Chintamani A, Tuteja A, Khandelwal R, Tandon M, Bamal R, Jain S, Narayan N, Srinivas S, Kumar Y (2011) Patient and provider delays in breast cancer patients attending a tertiary care centre: a prospective study. JRSM Short Rep 2(10):76

3. Ghumare SS, Cunningham JE (2007) Breast cancer trends in Indian residents and emigrants portend an emerging epidemic for India. Asian Pac J Cancer Prev 8(4):507-512

4. Chintamani A, Khandelwal R, Mittal A, Sai S, Tuteja A, Bhatnagar D, Saxena S (2007) Qualitative and quantitative dermatoglyphic traits in patients with breast cancer. BMC Cancer 7:44 